\title{
Postoperative high-flow nasal insufflation for obstructive sleep apnea: a potential therapeutic alternative or prudence needed?
}

\author{
Montserrat Diaz-Abad ${ }^{1}$, Paschalis Steiropoulos ${ }^{2}$, and Antonio M Esquinas ${ }^{3}$ \\ ${ }^{1}$ Department of Medicine, University of Maryland School of Medicine, Baltimore, MD, USA, ${ }^{2}$ Department of \\ Pneumonology, Medical School, Democritus University of Thrace, Alexandroupolis, Greece, ${ }^{3}$ Intensive Care Unit, \\ Hospital Morales Meseguer, Murcia, Spain
}

We read with great interest the article published in this journal titled "Postoperative use of high flow nasal insufflation for obstructive sleep apnea: a case series" by Gobindram et al. [1], which reported the results of the use of high-flow nasal insufflation (HFNI) in the postoperative period in patients diagnosed or suspected of having moderate-to-severe obstructive sleep apnea (OSA) with a history of continuous positive airway pressure (CPAP) intolerance.

This case series of three patients introduces an important indication of HFNI for OSA management in postoperative hypoventilation/apnea syndrome. However, we have the following considerations for a proper interpretation of the results and their application in the development of future studies:

\section{Limited Information on OSA Diagnosis and Prior CPAP Management}

While case 1 had a diagnosis of severe OSA, with an apneahypopnea index of $104 / \mathrm{h}$, there is no information on the timing

Corresponding author: Montserrat Diaz-Abad, M.D.

Division of Pulmonary and Critical Care Medicine, University of Maryland School of Medicine, Sleep Disorders Center, 100 North Greene Street Room 214, Baltimore, MD 21201, USA

Tel: +1-410-706-4771, Fax: +1-410-706-0345

Email:mdiaz@som.umaryland.edu

ORCID: https://orcid.org/0000-0001-8877-7313

Received: August 6, 2019

Revised: August 15, 2019.

Accepted: August 16, 2019.

Korean J Anesthesiol 2019 December 72(6): 622-623 https://doi.org/10.4097/kja.19337 or method of the diagnosis or the associated symptoms, such as excessive daytime sleepiness, which is crucial for CPAP management. Moreover, although it was reported that the patient was non-complaint with CPAP in the past, the cause, timing, and settings were not described; therefore, it is not clear that the patient was intolerant to CPAP. Additionally, the other two patients were only suspected of having OSA and did not have a definitive diagnosis. Therefore, the generalizability of the reported findings in patients with a confirmed diagnosis of OSA is limited. Furthermore, the protocol for CPAP initiation at home before the surgery in cases 2 and 3 was not described. Mask fitting and patient education are recommended for initiation of CPAP therapy at home in patients with OSA, and it is important to troubleshoot any problems to optimize the acceptance of the therapy.

\section{Breathing Pattern, Evaluation, Hypoventilation Apneas/Hypopneas, Postoperative Period, and Monitoring}

The information on respiratory rate in the postoperative period was provided, but it seems hypoventilation and/or apneas or hypopneas were not monitored, as described by Zhang et al. [2]. It would be useful to consider monitoring these events with methods such as capnography, which can confirm the stability of ventilation $[3,4]$, both before and during therapy with HFNI. This is of great importance, especially in patients with comorbidities, as in case 2 .

\section{Surgery/Indications}

Two patients who underwent tonsillectomy were reported.

(c) This is an open-access article distributed under the terms of the Creative Commons Attribution Non-Commercial License (http://creativecommons.org/ licenses/by-nc/4.0/), which permits unrestricted non-commercial use, distribution, and reproduction in any medium, provided the original work is properly cited. 
However, the indications for tonsillectomy or its association with OSA were not mentioned. While increased airway collapsibility and worsening of OSA could be a first-night effect of residual anesthesia [5], the removal of a large portion of the tonsil could relieve airway obstruction in some patients. One cannot know if the observed improvement in OSA in the postoperative phase resulted from the tonsillectomy or HFNI. This is an important factor to consider. We recommend further studies including patients who have not undergone airway surgeries, thereby eliminating the potential confounding factor of postoperative airway edema.

\section{HFNI Flow and Oxygenation Settings}

The protocol for the implementation and titration of HFNI was not fully described, including the initial and targeted peripheral capillary oxygen saturation values and the criteria for choosing the settings selected in each case. Furthermore, it needs to be elucidated whether the same HFNI flow and oxygenation would be effective in different sleep stages and body positions or would they need to be adjusted throughout the night.

In conclusion, it is critical to prevent perioperative complications in patients with OSA. With this case series, the authors introduce a new and important indication for HFNI. We believe that our comments and suggestions might be useful for the development of future studies.

\section{Conflicts of Interest}

No potential conflict of interest relevant to this article was reported.

\section{Author Contributions}

Montserrat Diaz-Abad (Formal analysis; Writing-review \& editing)

Paschalis Steiropoulos (Formal analysis; Writing-review \& editing)

Antonio M Esquinas (Conceptualization; Formal analysis; Supervision; Writing-original draft)

\section{ORCID}

Montserrat Diaz-Abad, https://orcid.org/0000-0001-8877-7313

Paschalis Steiropoulos, https://orcid.org/0000-0001-7121-6253

Antonio M Esquinas, https://orcid.org/0000-0003-0571-2050

\section{References}

1. Gobindram A, Singh PA, Quek KH. Postoperative use of high flow nasal insufflation for obstructive sleep apnea-a case series. Korean J Anesthesiol 2019; 72: 610-3.

2. Zhang X, Kassem MA, Zhou Y, Shabsigh M, Wang Q, Xu X. A brief review of non-invasive monitoring of respiratory condition for extubated patients with or at risk for obstructive sleep apnea after surgery. Front Med (Lausanne) 2017; 4: 26.

3. Voscopoulos C, Ladd D, Campana L, George E. Non-invasive respiratory volume monitoring to detect apnea in post-operative patients: case series. J Clin Med Res 2014; 6: 209-14.

4. Burton JH, Harrah JD, Germann CA, Dillon DC. Does end-tidal carbon dioxide monitoring detect respiratory events prior to current sedation monitoring practices? Acad Emerg Med 2006; 13: 500-4.

5. De A, Waltuch T, Gonik NJ, Nguyen-Famulare N, Muzumdar H, Bent JP, et al. Sleep and breathing the first night after adenotonsillectomy in obese children with obstructive sleep apnea. J Clin Sleep Med 2017; 13: 805-11. 